Research Article

\title{
The Influence of the Motor Traction Vibration on Fatigue Life of the Bogie Frame of the Metro Vehicle
}

\author{
Qiushi Wang, Jinsong Zhou (D, Dao Gong, Tengfei Wang, Jiangxue Chen, Taiwen You, \\ and Zhanfei Zhang
}

Institute of Rail Transit, Tongji University, Shanghai 201814, China

Correspondence should be addressed to Jinsong Zhou; jinsong.zhou@tongji.edu.cn

Received 6 January 2020; Revised 25 June 2020; Accepted 4 October 2020; Published 23 October 2020

Academic Editor: F. Viadero

Copyright ( $\odot 2020$ Qiushi Wang et al. This is an open access article distributed under the Creative Commons Attribution License, which permits unrestricted use, distribution, and reproduction in any medium, provided the original work is properly cited.

\begin{abstract}
During the service life of metro vehicles, the cracks frequently appear at the root of motor seats. This indicates the previous antifatigue designs are unable to cover the actual operating environment. Some individual loads, such as motor vibration, have been ignored or wrongly understood, which leads to the occurrence of local insufficient fatigue life of the frame. To illustrate the influence of the motor vibration on the fatigue life of the bogie frame, a metro vehicle was taken as an example: first, the precise finite element model of the frame was established, and its correctness was verified; then, the vibration characteristics of the frame were analyzed by sweep frequency calculation; and finally, considering the vibration acceleration signal of the motor measured on a metro line as the excitation, the influence of the random vibration on the fatigue life of the frame under traction and idle running conditions was compared and analyzed by solving the power spectral density of the dynamic stress response at the weak fatigue nodes of the structure. The results show that the energy of the vibration in the frame is mainly concentrated in modes 6 and 7 , which are excited by the motor transverse and vertical vibration, respectively, and contribute a lot to the fatigue damage of the frame; the fatigue life of the vital positions of the frame under the traction condition significantly reduces compared to the idle running condition; and the contribution of the low-amplitude vibration above $300 \mathrm{~Hz}$ to the fatigue damage can be ignored. At last, the importance of the influence of the motor traction vibration on the fatigue life should be fully considered in the metro vehicle design was proposed.
\end{abstract}

\section{Introduction}

During the operation, metro vehicles were excited by various loads. These loads not only come from track irregularities but also the inside as well, such as traction motor vibration, within a wide frequency range [1-3]. Some studies mentioned that many fatigue cracks appeared around the area near to the traction motor seats during their service life, which was thought to be related to the local elastic vibration $[4,5]$. There are only a few research studies about motor vibration fatigue on the metro bogie frame. Some studies show that (1) the vertical vibration of the frame suspension traction motor has an evident impact on the frame fatigue strength [6]; (2) decoupling optimization of the motor suspension system can effectively improve the vibration isolation to reduce the fatigue damage of the motor seat
$[7,8]$. The above studies have confirmed the importance of the influence of the motor vibration on the fatigue strength of the frame from the obverse and reverse, but have not been studied in detail.

Figure 1 shows a complete time-domain signal of the motor acceleration vibration from station $\mathrm{A}$ to station $\mathrm{B}$ in a city metro line. It can be seen that the metro vehicle has experienced "traction-idle running-brake" conditions successively, and the traction condition includes the starting condition and rated condition. It is worth noting that, in a large number of previous fatigue designs and calculations, the starting condition was often considered as an extraordinary condition, which leads to the influence of the starting condition on the fatigue of the frame which is omitted [9-11]. For this question, EN13749-2005 (Appendix D in [12]) has stated that it is necessary to consider the number of 


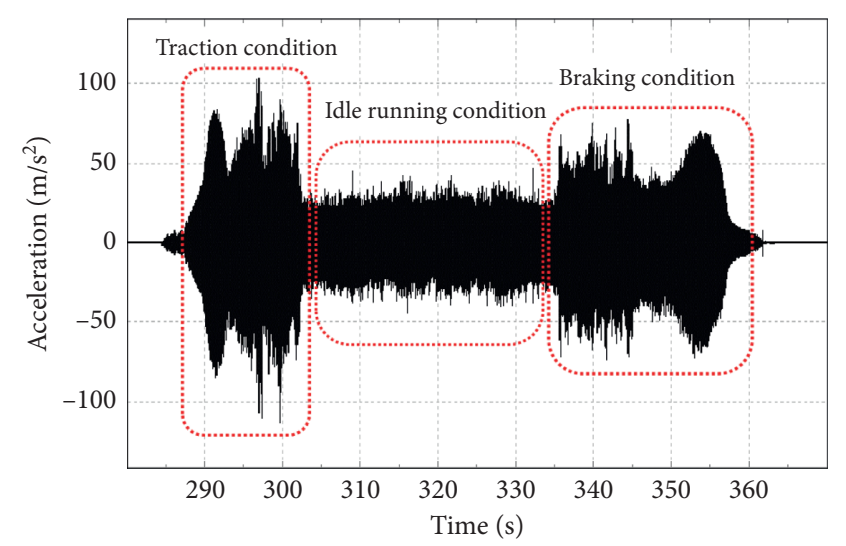

Figure 1: Vertical acceleration vibration signal of a metro motor from station A to station $\mathrm{B}$.

applications of these loads in any fatigue damage analysis [12]. For such frequently started railway vehicles as metro, the ratio of the starting condition to their entire service life is significantly different from the high-speed EMU in the same service mileage. Besides, according to the existing research, high-amplitude loads often contribute a lot to the fatigue damage [13]. However, the starting conditions often cause large-amplitude loads. Therefore, the research on the influence of the motor traction vibration (including starting condition) on the fatigue life of the bogie frame in metro vehicles has practical significance.

The vibration under the braking condition is not caused by motor traction, so it will not be discussed in this research. Some studies show that the longitudinal vibration of the motor has contributed a little to the fatigue damage of the frame, so the study on the influence of the longitudinal motor vibration will not be performed in this paper [6].

The outline of this paper is as follows: first, the modal shape and the main frequency of the frame were calculated by the finite element method (FEM), and the FEM results were compared with the experimental modal analysis (EMA) results to verify the correctness of the model. Then, the dynamic characteristics of the bogie frame were analyzed through sweep frequency calculation. Finally, by fully considering the contribution of the high-frequency vibration to the fatigue damage of the frame, the fatigue life of the bogie frame was calculated separately with the motor vibration under traction and idle running conditions within $1300 \mathrm{~Hz}$.

\section{Establishment and Verification of the Model}

2.1. Finite Element Calculation. The finite element model of the metro bogie frame was built by HyperMesh 19.0, as shown in Figure 2. The motor seats, gearbox seats, and parts of the midbeam were meshed as three-dimensional solid elements. The side beams, ribs, etc., were meshed as twodimensional shell elements. The numbers of solid and shell elements are 286,492 and 102,839, and the numbers of nodes for the solid and shell are 63,996 and 101,933. The coordinate system is defined as follows: the forward direction of the vehicle is the $X$-axis; the $Y$-axis is parallel with the track

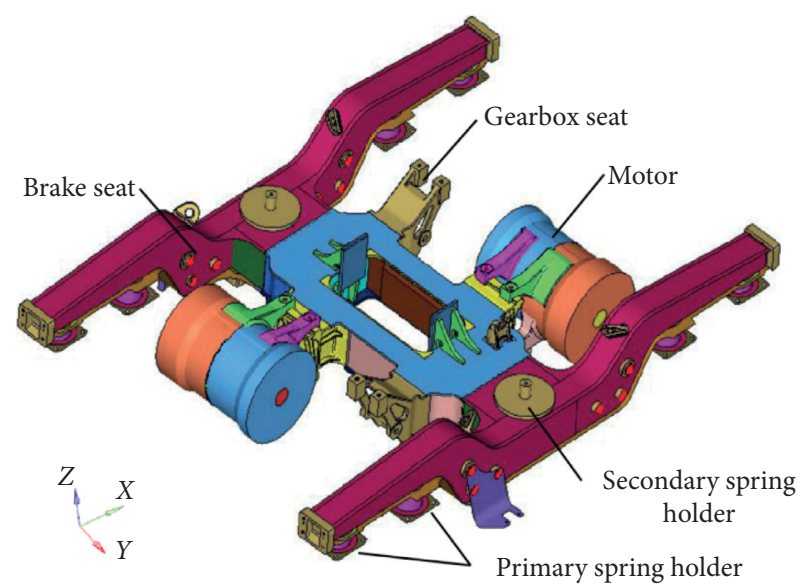

FIgURE 2: Finite element model of the bogie frame.

plane and perpendicular to the $X$-axis; and the $Z$-axis is perpendicular to the track plane. Rigid connections were established between the motor and the motor mounting base, and the completely free modes of the frame without the unconstrained condition were calculated.

According to the modal analysis theory of structure vibration, before the modal verification, the bogie system's natural modal characteristics need to be solved. The damping ratio is generally $0.01 \sim 0.1$ for the bogie frame structure. Therefore, the influence of the damping ratio on the natural frequency can be ignored. The fundamental equation of an undamped free vibration system can be expressed as

$$
[M]\{\ddot{u}\}+[K]\{u\}=0,
$$

where $[M]$ represents the mass matrix, $[K]$ represents the stiffness matrix, and $\{u\}$ represents the displacement vector. Assume that the special solution of formula (1) is as follows:

$$
\{u\}=\{\varphi\} \sin \omega\left(t-t_{0}\right),
$$

where $\{\varphi\}$ represents an $n$-order eigenvector, $\omega$ represents the eigenvalue corresponding to $\{\varphi\}$, and $t_{0}$ represents a constant determined by the initial conditions.

Solve (1) by substituting (2), and get analytical solution (3). The calculation results are $\left(\omega_{1}^{2}, \varphi_{1}\right),\left(\omega_{2}^{2}, \varphi_{2}\right), \ldots,\left(\omega_{n}^{2}, \varphi_{n}\right)$. The parameters $\omega_{1}, \omega_{2}, \ldots, \omega_{n}$ represent the eigenvalues of the system. The eigenvectors $\left\{\varphi_{1}\right\},\left\{\varphi_{2}\right\}, \ldots,\left\{\varphi_{n}\right\}$ represent the natural modes of the system:

$$
[K]\{\varphi\}-\omega^{2}[M]\{\varphi\}=0 .
$$

2.2. Experimental Modal Test. The EMA (experimental modal analysis) of the frame was carried out to verify the simulation model's correctness. The hammering method and SISO test method were used to perform the EMA on the bogie frame. The frame was not equipped with wheelsets, gearboxes, and other components. With suspending by a crane's hoisting rope, it can be considered as a completely free and unconstrained condition, as shown in Figure 3. 


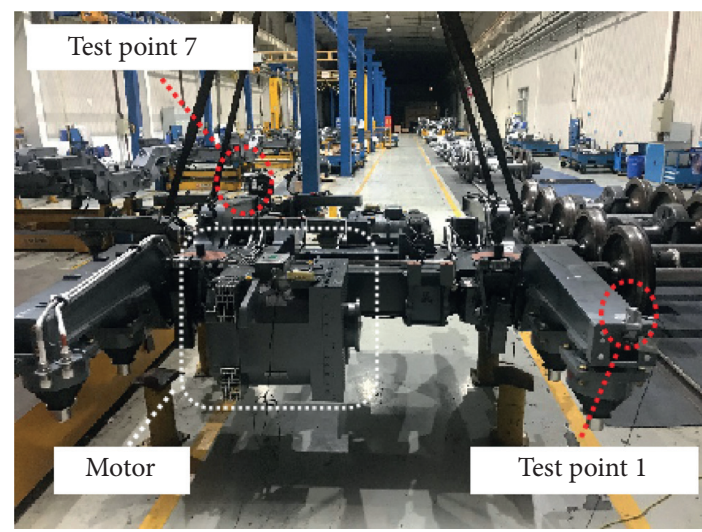

Figure 3: Experimental modal test for the bogie frame.

As shown in Figure 4, positions 1 and 7, with the threedirection piezoelectric acceleration sensors stuck here, are the vibration response test points. The hammering points were arranged at the other 30 vital geometric positions of the frame. The signals caused by the medium force hammer were collected through the CPCI box.

2.3. Analysis and Verification. The FEM model (Figure 2) was imported into commercial software. The block Lanczos algorithm was used to calculate the modal shape and the main frequency of the bogie frame with the frequency below $100 \mathrm{~Hz}$. The modal shapes are shown in Figure 5, with the theoretical calculation results on the left and the modal test results on the right.

The MAC function (modal assurance criterion) was used to describe the correlation between theoretical and measured modal shapes, as shown in formula (4). The closer the MAC's calculated value is to 1 , the higher the two modal shapes' correlation degree is. The calculation results of the correlation are shown in Table 1.

$$
\left[\operatorname{MAC}\left(\left\{\phi_{e}\right\}_{j},\left\{\phi_{f}\right\}_{k}\right)\right]=\frac{\left(\left\{\phi_{e}\right\}_{j}^{T}\left\{\phi_{f}\right\}_{k}\right)^{2}}{\left\{\phi_{e}\right\}_{j}^{T}\left\{\phi_{e}\right\}_{j}\left\{\phi_{f}\right\}_{k}^{T}\left\{\phi_{f}\right\}_{k}},
$$

where $\left\{\varphi_{e}\right\}_{j}$ represents the $j$-th modal shape vector of the finite element analysis results and $\left\{\varphi_{f}\right\}_{k}$ represents the $k$-th modal shape vector of the experimental modal analysis results.

All the relative errors between the FEM results and EMA results for natural frequency corresponding to each mode are less than $7.50 \%$ (the maximum error is $7.37 \%$ ), which can be accepted in engineering, as shown in Table 1 . Therefore, the accuracy of the finite element model has been verified.

\section{Random Vibration Analysis}

Stress (strain) is always essential data for fatigue assessment and life prediction. There are two kinds of methods to calculate the stress in the bogie frame: static stress method and dynamic stress method. For example, UIC615-4 [14] and EN13749, the standards for fatigue strength design of the bogie frame, are the static stress analysis methods. Although the methods are practical and straightforward, the effect of

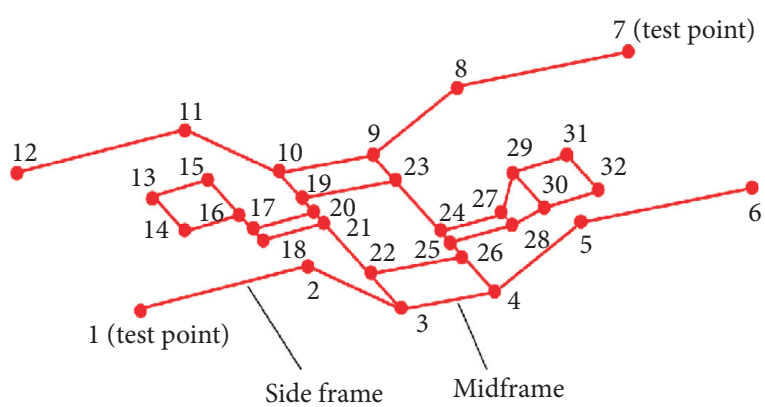

FIGURE 4: Layout of test points.
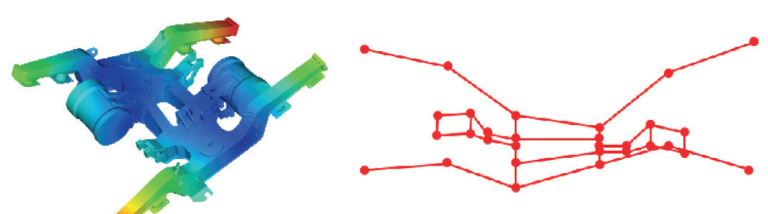

Frequency $=56.066 \mathrm{~Hz}$

Frequency $=52.680 \mathrm{~Hz}$

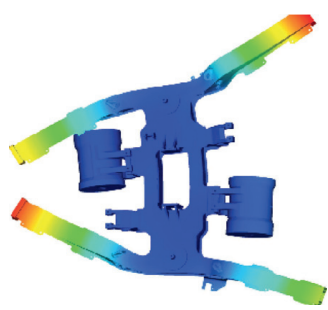

Frequency $=64.972 \mathrm{~Hz}$
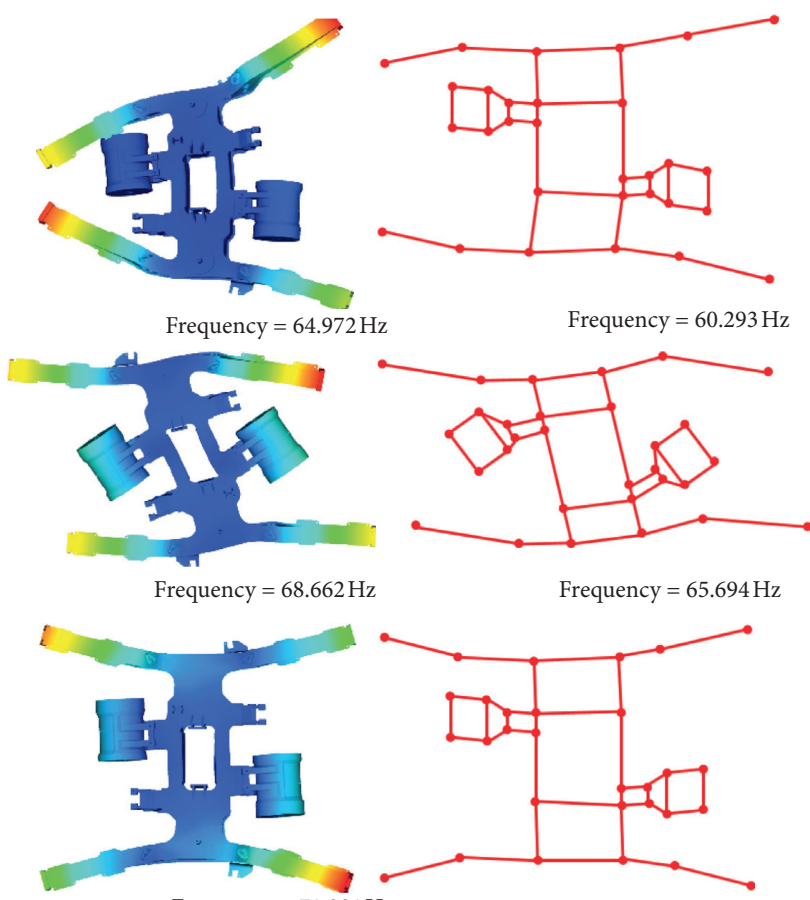

Frequency $=71.291 \mathrm{~Hz}$
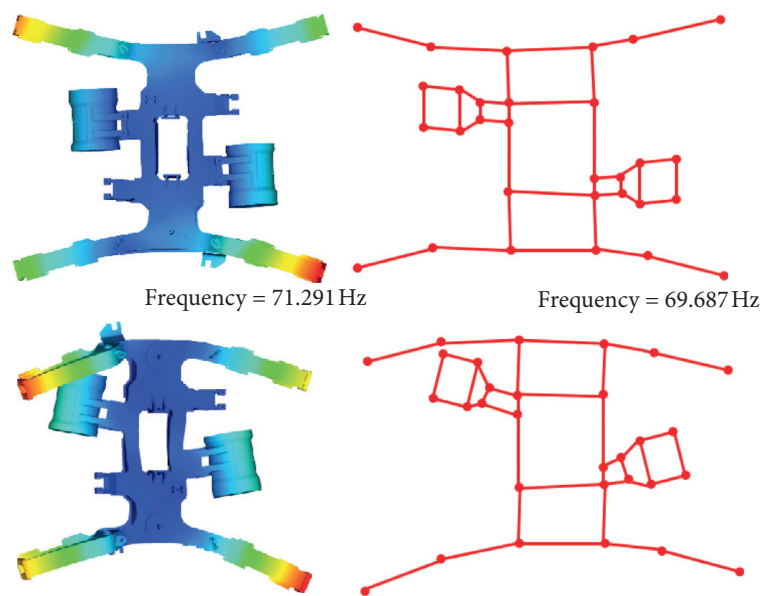

Frequency $=78.885 \mathrm{~Hz}$

Frequency $=73.072 \mathrm{~Hz}$
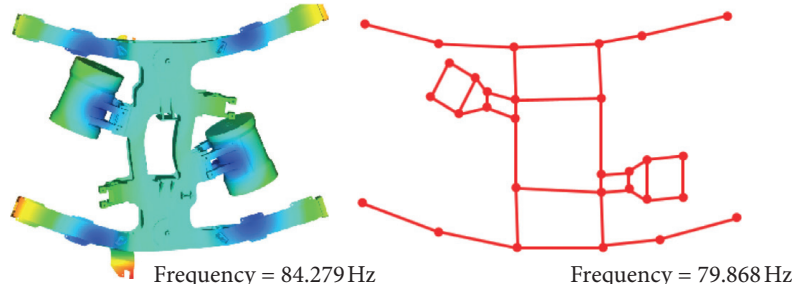

Figure 5: Comparison of modal shapes of FEM and EMA results. 
TABLE 1: Modal analysis of FEM and EMA results.

\begin{tabular}{|c|c|c|c|c|c|c|}
\hline \multicolumn{2}{|c|}{ FEA modal result } & \multicolumn{2}{|c|}{ Test modal result } & \multirow{2}{*}{ Error (\%) } & \multirow{2}{*}{ MAC } & \multirow{2}{*}{ Modal shape } \\
\hline Modal order & Frequency $(\mathrm{Hz})$ & Modal order & Frequency $(\mathrm{Hz})$ & & & \\
\hline 1 & 49.370 & 1 & 47.010 & 4.78 & 0.96 & Whole shearing deformation \\
\hline 2 & 56.066 & 2 & 52.680 & 6.04 & 0.94 & Whole twisting deformation \\
\hline 3 & 64.972 & 3 & 60.293 & 7.20 & 0.94 & Side beam bending as "V" type \\
\hline 4 & 68.662 & 4 & 65.694 & 4.32 & 0.95 & Side beam bending as "diamond" type \\
\hline 5 & 71.291 & 5 & 69.687 & 2.25 & 0.98 & Side beam lateral bending on the reverse direction \\
\hline 6 & 78.885 & 6 & 73.072 & 7.37 & 0.96 & Side beam lateral bending in the same direction \\
\hline 7 & 84.279 & 7 & 79.868 & 5.23 & 0.95 & Side beam lateral bending in the same direction \\
\hline
\end{tabular}

resonance fatigue has been ignored. The random vibration analysis is the method based on the dynamic stress, mainly including the quasi-static method, time-domain method, and frequency-domain method:

(1) The quasi-static method is generally used to analyze the engineering problem that the loading frequency is far away from the main frequency of the structure. The dynamic stress response of each key point is obtained by using the quasi-static response coefficient and random load spectrum.

(2) The time-domain method is based on the input of the time-domain signal of the random load and obtains the dynamic stress response of each point through the mode superposition method or direct integration method. While dealing with a long time-domain signal, the method will spend a lot of running time and cost a lot of storage space. Sometimes, it cannot be even solved out.

(3) The frequency-domain method is a widely used analysis method. The time-domain signal is transformed into the power spectral density (PSD) in the frequency domain with the Fourier transform. As the input, the PSD for displacement, velocity, or acceleration is used to calculate the dynamic stress response based on the established transfer function.

Because of the long time-domain signal of the whole metro line, about 24.5 kilometres, the time-domain method cannot be applied to complete the analysis. Therefore, the frequencydomain method was used in this analysis to solve the dynamic stress response of several nodes of the bogie frame.

3.1. Power Spectral Density (PSD) Function. The vibration generated by the operation of metro vehicles can be regarded as a wide-sense stationary random process (WSRP). The autocorrelation function $R_{q q}(\tau)$ of the time-domain signal $q(t)$ depends on the time difference $\tau=t_{2}-t_{1}$. The autocorrelation function $R_{q q}(\tau)$ is defined as equation (5). Besides, $R_{q q}(\tau)$ and power spectrum density $S_{q q}(\omega)$ are a Fourier-transform pair, defined as equation (6):

$$
\begin{aligned}
& R_{q q}(\tau)=\int_{-\infty}^{+\infty} q_{1}\left(t_{1}\right) q_{2}\left(t_{2}\right) p\left(q_{1}, q_{2} ; t_{1}, t_{2}\right) \mathrm{d} q_{1} \mathrm{~d} q_{2}, \\
& S_{q q}(\omega)=\frac{1}{2 \pi} \int_{-\infty}^{+\infty} R_{q q}(\tau) e^{-j \omega \tau} \mathrm{d} \tau .
\end{aligned}
$$

Through the piezoelectric acceleration sensor (Figure 6(a)) and CPCI box (Figure 6(b)), the vertical and lateral acceleration vibration signals of the motor are measured on a metro line. Since the vibration of the motor measured on a metro operation line comes from the traction and idle running conditions together (Figure 1), the motor's vibration acceleration signals in the traction condition and the idle running condition were selected, respectively. And then, the power spectrum density (PSD) of acceleration obtained by Fourier transform is shown in Figure 7.

Power spectrum density (PSD) function represents the distribution of energy in the frequency domain in a random process [15]. As shown in Figure 7, the main vibration of the motor is the vertical vibration because the amplitude of vertical vibrations is more than ten times of the transverse vibration. The maximum value of the vertical acceleration peak is $5.84\left(\mathrm{~m} / \mathrm{s}^{2}\right)^{2}$ under the traction condition. The maximum value of the vertical acceleration peak is $3.22(\mathrm{~m} /$ $\left.s^{2}\right)^{2}$ under the idle running condition. It shows that the vibration of the motor under the traction condition is enhanced.

Besides, except for the energy distribution within $500 \mathrm{~Hz}$ under the traction condition, there are still visible peak signals at the frequency domain of $1000 \mathrm{~Hz} 2500 \mathrm{~Hz}$, as shown in Figure 7(a). Therefore, the influence of the highfrequency vibration of the motor on the fatigue strength of the frame was considered in this analysis. Because of the limited storage capacity and calculation capacity of the computer, the signals with frequency less than $1300 \mathrm{~Hz}$ were intercepted for analysis.

\subsection{Theoretical Analysis of the Random Vibration.} According to the mechanical vibration theory, there are two methods which are used to take acceleration as the input in random vibration analysis: fixed-point excitation method and relative motion method. The fixed-point excitation needs to convert the acceleration into force or torque and then apply it to the structure. However, there is no better method yet to identify the absolute excitation force of a rigidly connected structure.

The relative motion method requires applying a full constraint on the load excitation point and applying an acceleration field in the whole system, which is equivalent to the load excitation point which vibrates at the same acceleration of the applied acceleration field in the system. 


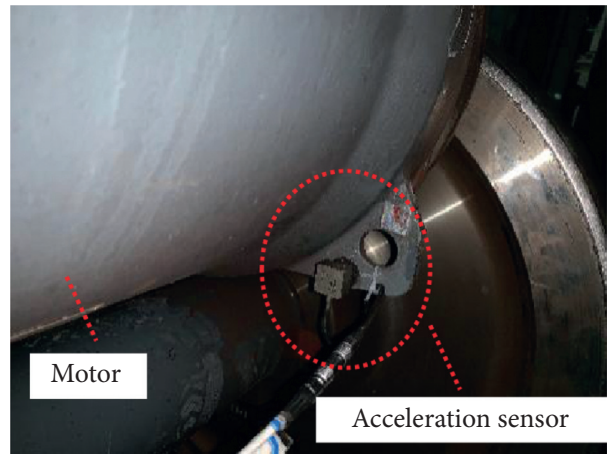

(a)

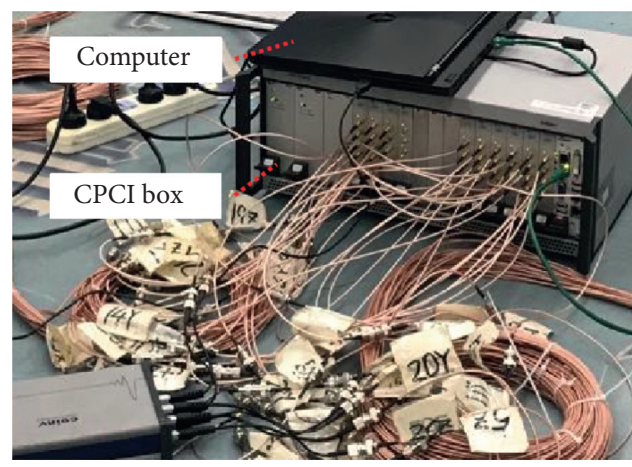

(b)

Figure 6: (a) Motor test point. (b) CPCI box and computer.

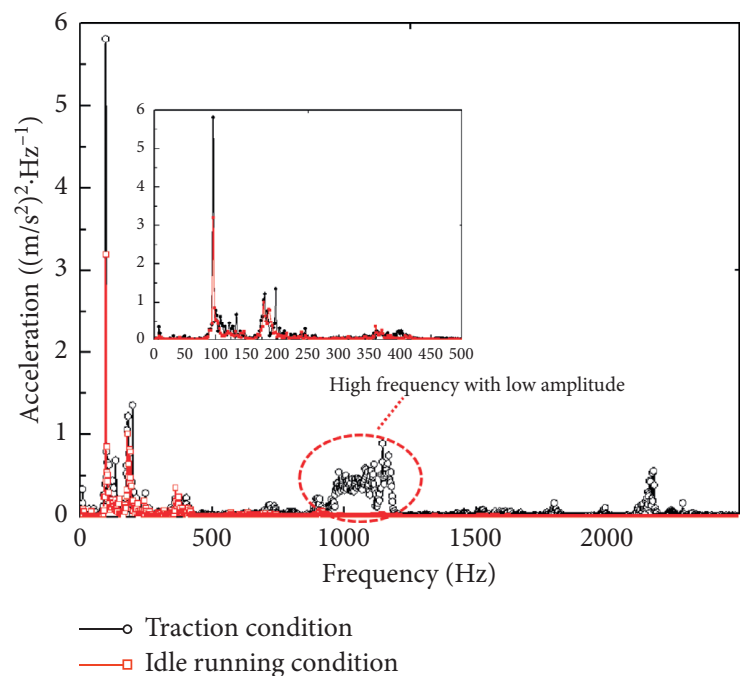

(a)

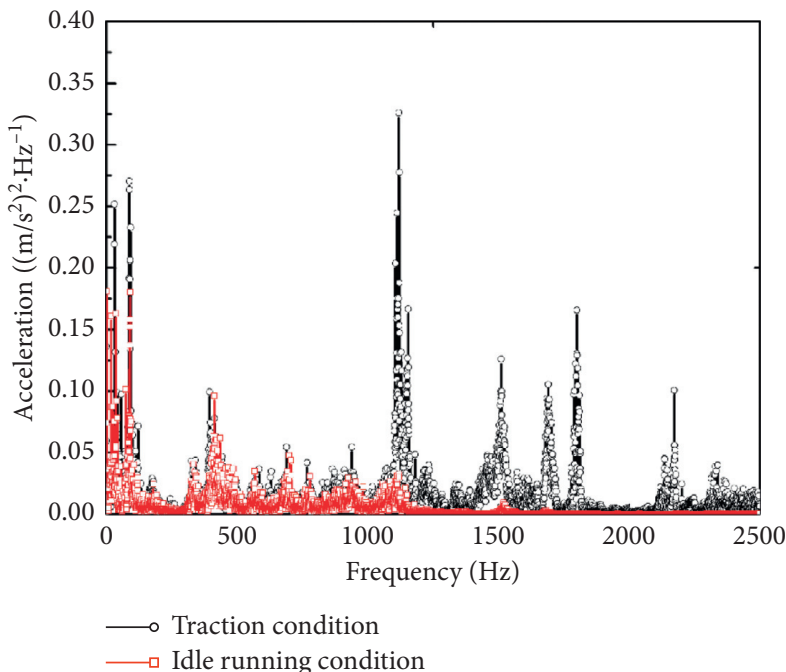

(b)

Figure 7: (a) Acceleration PSD of the motor (vertical). (b) Acceleration PSD of the motor (horizontal).

In this paper, the relative motion method (Figure 8) was used to analyze the influence of the motor vibration on the frame's fatigue life. The measured motor acceleration vibration signal was directly used as the input condition, and $X, Y$, and $Z$ direction constraints were applied on the center of mass of the motor. The other constraints were released at the same time. The theoretical derivation process is as follows.

The single-degree-of-freedom system is shown in Figure 8. Its dynamic differential equation can be written as

$$
m \ddot{y}+c(\dot{y}-t \dot{x})+k(y-x)=0,
$$

where $y$ represents the absolute displacement of the system response, $x$ represents the displacement of the reference position, and $z=y-x$ represents the relative displacement between them. Equation (7) can be written as equation (8). Therefore, the dynamic differential equation of the multiple degrees of freedom system can be written as equation (9):

$$
\begin{aligned}
m \ddot{z}+c \dot{z}+k z & =-m \ddot{x}, \\
{[M]\{\ddot{z}\}+[C]\{\dot{z}\}+[K]\{z\} } & =-[M]\{E\} \ddot{x}(t) .
\end{aligned}
$$

According to the mechanical vibration theory, the calculation results of the response of the MDOF system can be expressed as

$$
\left[S_{z z}(\omega)\right]=[H]^{*}\left[S_{x x}(\omega)\right][H]^{T} .
$$

And the spectral matrix relationship between the input and the response can be expressed as equations (11) and (12):

$$
\begin{aligned}
& {\left[S_{x z}(\omega)\right]=\left[S_{x x}(\omega)\right][H]^{T},} \\
& {\left[S_{z x}(\omega)\right]=[H]^{*}\left[S_{x x}(\omega)\right] .}
\end{aligned}
$$

However, the solution $z$ of equation (8) is the relative displacement. The absolute displacement $y$ is the 


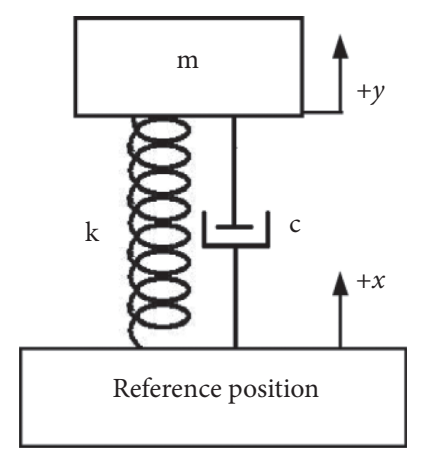

FIGURE 8: Dynamic model of the relative motion method.

superposition of the relative displacement $z$ and the reference position displacement $x, y(t)=z(t)+x(t)$. The autocorrelation function of absolute displacement $y(t)$ can be easily derived as

$$
\begin{aligned}
R_{y y}(\tau)= & E[Y(t) \cdot Y(t+\tau)]=E[(Z(t)+X(t)) \\
& \cdot t(Z(t+\tau)+X(t+\tau))] \\
= & R_{z z}(\tau)+R_{z x}(\tau)+R_{x z}(\tau)+R_{x x}(\tau) .
\end{aligned}
$$

According to equation (6), the absolute displacement power spectral density of the system can be expressed as

$$
\left[S_{y y}(\omega)\right]=\left[S_{z z}(\omega)\right]+\left[S_{z x}(\omega)\right]+\left[S_{x z}(\omega)\right]+\left[S_{x x}(\omega)\right],
$$

where $[M],[C]$, and $[K]$ represent the mass, damping, and stiffness matrices of the system structure, $\{E\}$ represents the indicating vector of the inertial force, and $[H]$ represents the frequency response function matrix.

\subsection{Analysis of Vibration Characteristics Based on Sweep} Frequency Calculation. The random vibration analysis module of finite element commercial software was used to analyze the frame's structural vibration characteristics. According to the theory of the relative motion method, the center of mass of the motor is constrained in $X, Y$, and $Z$ directions, and the unit-amplitude-sine acceleration field in $+Y$ and $+Z$ directions was applied to the system to perform the sweep frequency calculation. The range of the sweep frequency is set at $0 \sim 1300 \mathrm{~Hz}$.

Figure 9 shows the calculation results of the root mean square (RMS) cloud diagram for the dynamic stress. The three nodes in the most concentrated area of $\sigma_{\mathrm{RMS}}$ are selected as the focus nodes: P1 (number 152331), P2 (number 164533), and P3 (number 208674), as shown in Figure 9(b). $\mathrm{P} 1$ is located at the motor seat body, $\sigma_{\mathrm{RMS}}=136.9 \mathrm{MPa}$. $\mathrm{P} 2$ is located at the root of the motor seat base (at the fillet of the midbeam), $\sigma_{\mathrm{RMS}}=108.1 \mathrm{MPa}$. P3 is located in the middle of the bottom plate of the side beam (at the fillet with the midbeam), $\sigma_{\mathrm{RMS}}=79.7 \mathrm{MPa}$. The above three positions are also consistent with where the actual fatigue cracks have appeared. Therefore, the three nodes are taken as the later research object.
The dynamic stress responses of $\mathrm{P} 1, \mathrm{P} 2$, and $\mathrm{P} 3$ within $250 \mathrm{~Hz}$ are extracted for analysis, as shown in Figure 10. Mode $2(56.066 \mathrm{~Hz})$ and mode $7(84.279 \mathrm{~Hz})$ of the frame generate large resonance due to the vertical vibration of the motor. Mode $6(78.885 \mathrm{~Hz})$ of the frame generates large resonance due to the motor's transverse vibration. Besides, the closer the node is to the motor, the more resonant modes will occur (number of resonance frequencies: P1 $>$ P2 $>$ P3). This proved that the influence of the motor traction vibration on the frame is local elastic resonance.

3.4. Analysis of the Random Vibration Based on the Measured Signal. Considering the lateral and vertical vibration acceleration signal of the motor measured on a metro line as the excitation (Figure 7), the relative motion method was used to solve the dynamic stress power spectral density of the random vibration response of the frame in actual operation. The technical route is as follows:

(1) The center of mass of the motor is constrained in $X$, $Y$, and $Z$ directions in the frame finite element model.

(2) The measured acceleration vibration power spectral density of the motor is decomposed. The frequency range is set at $0 \sim 1300 \mathrm{~Hz}$, and the frequency step is set as $\Delta \omega=0.1 \mathrm{~Hz}, \omega_{j}=\omega_{\min }+j \bullet \Delta \omega(j=0,1,2, \ldots, n)$.

(3) Several frequency-domain PSD signals which come from Figure 7 are taken as the acceleration field in $+Y$ and $+Z$ directions to apply to the frame system at the same time.

(4) The dynamic stress response power spectral density of the nodes is calculated after the modal expansion.

The dynamic stress responses of $\mathrm{P} 1, \mathrm{P} 2$, and $\mathrm{P} 3$ are extracted, and the double logarithmic spectrum is drawn for analysis, as shown in Figure 11. The calculation results' data are organized, as shown in Table 2. The main frequencies of the key nodes (P1, P2, and P3) under the traction and idle running conditions are the same. The dynamic stress response amplitude of mode 7 and mode 6 of the model is more significant than others (Table 2). The results show that the vibration energy of the frame is mainly concentrated in mode $7(84.279 \mathrm{~Hz})$ and mode $6(78.885 \mathrm{~Hz})$, which contribute a lot to the fatigue damage of the frame.

According to the analysis of vibration characteristics given in Section 3.3, mode $7(84.279 \mathrm{~Hz})$ is excited by the motor's vertical vibration; mode $6(78.885 \mathrm{~Hz})$ is excited by the motor's transverse vibration. Even though the transverse vibration amplitude of the motor is much smaller than the vertical vibration (Figure 7(b)), it still has a high impact on the frame's dynamic stress response. Therefore, in the fatigue strength design and assessment of the frame, the influence of the transverse vibration of the motor on the fatigue life of the frame should not be ignored.

\section{Fatigue Assessment in the Frequency Domain}

The fatigue assessment method based on frequency-domain statistics was used to calculate the vibration fatigue life. Some 


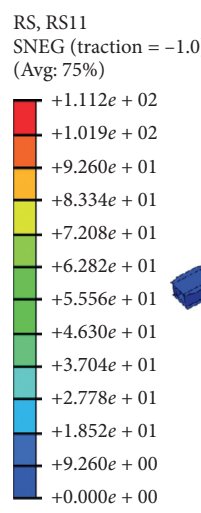

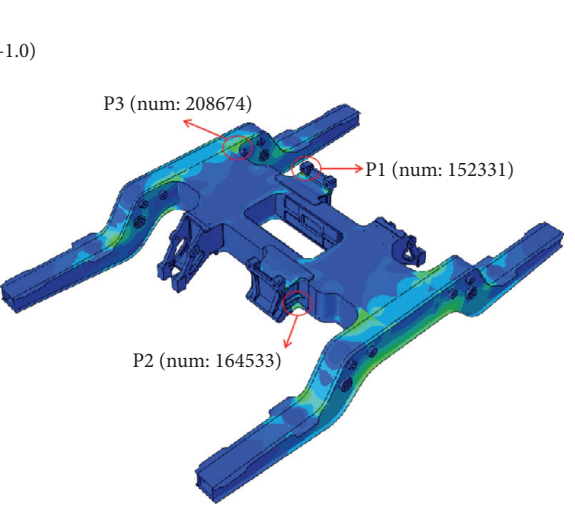

(a)
RS, RS11

SNEG $($ traction $=-1.0$ )

(Avg: 75\%)

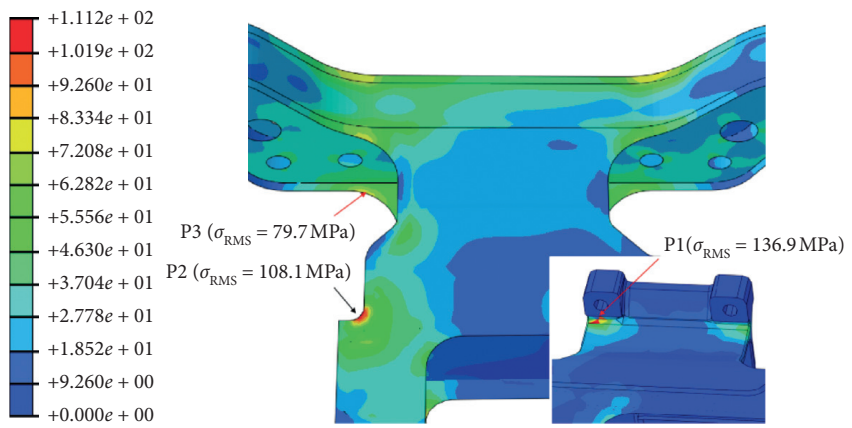

(b)

Figure 9: (a) RMS stress cloud diagram for the whole bogie frame (MPa). (b) RMS stress cloud diagram for the local part (MPa).

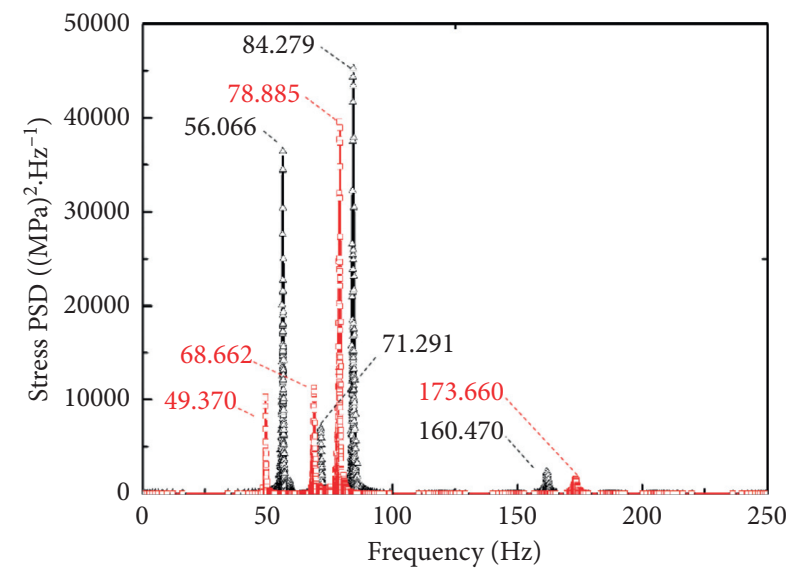

$\triangle \mathrm{Z}$ direction

$-\square-$ Y direction

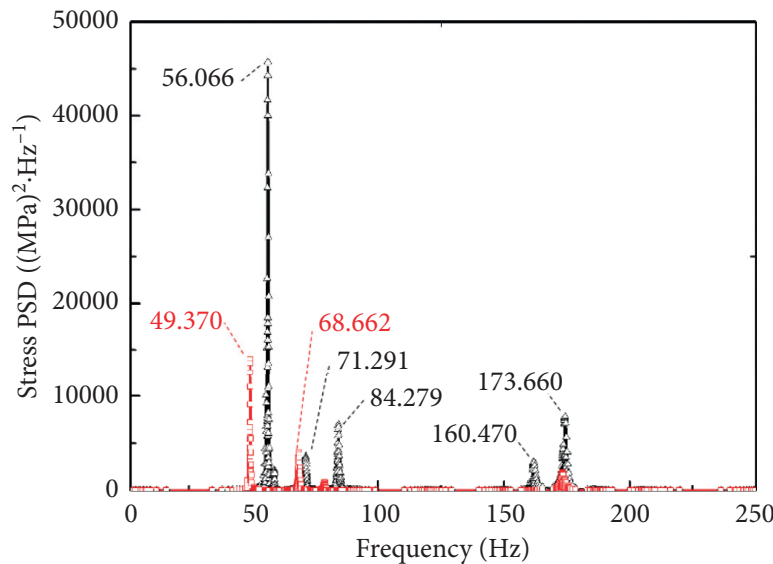

$-\checkmark \mathrm{Z}$ direction

$\neg-\mathrm{Y}$ direction

(b)

(a)

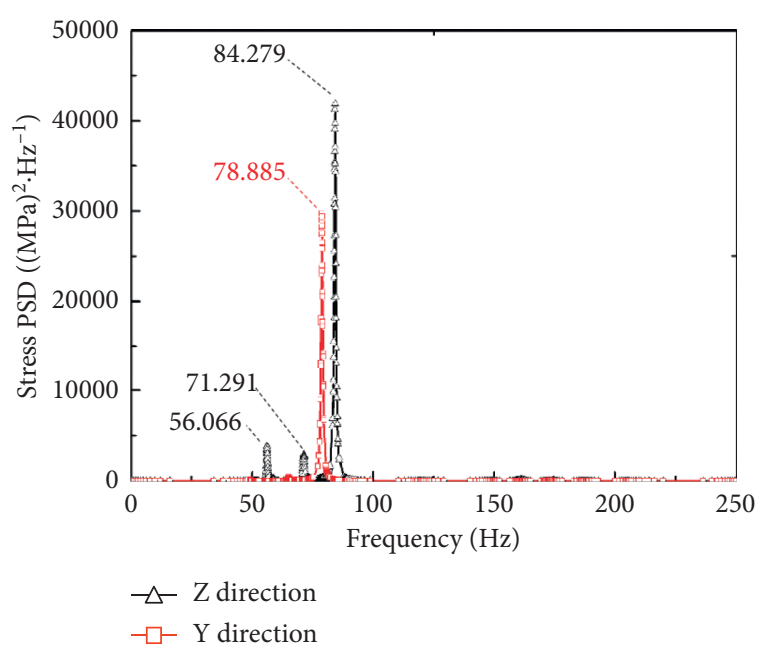

(c)

Figure 10: (a) Dynamic stress response of P1. (b) Dynamic stress response of P2. (c) Dynamic stress response of P3. 


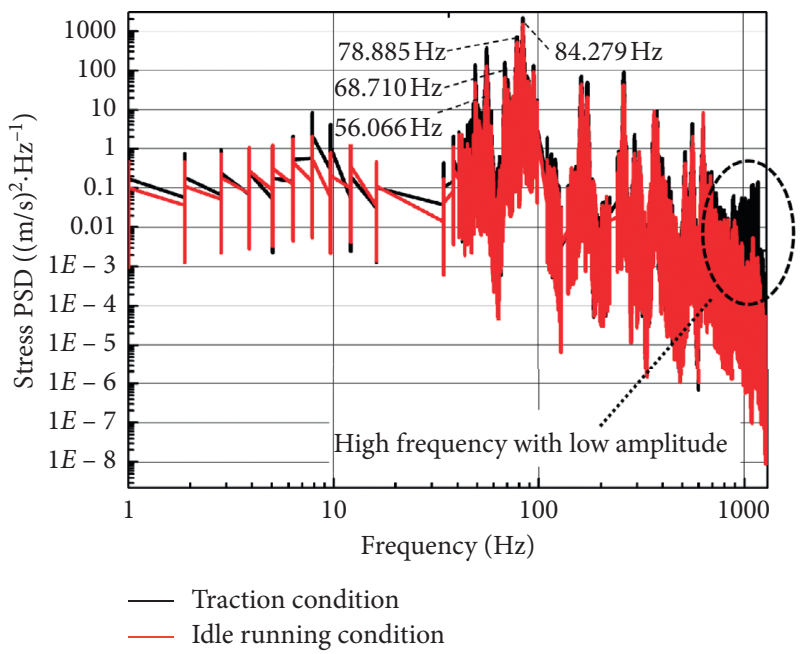

(a)

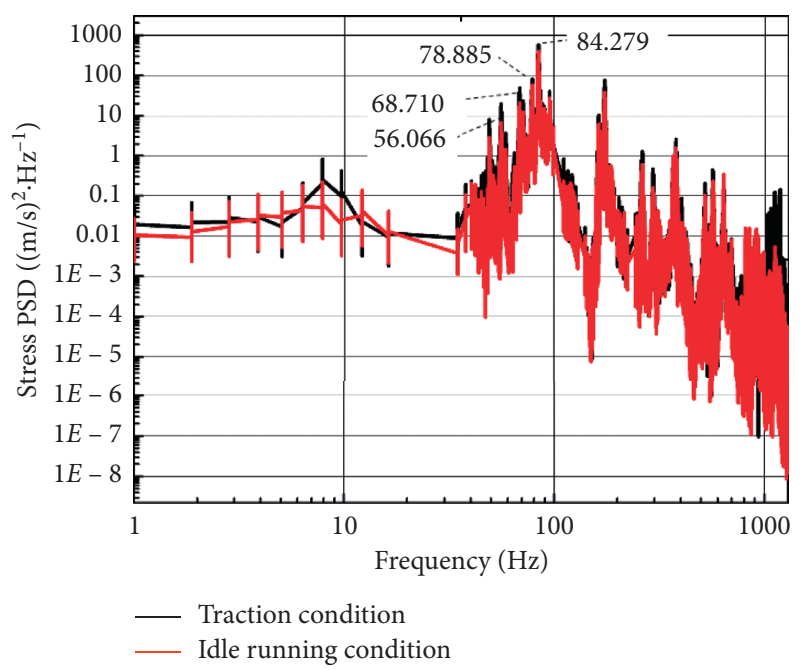

(b)

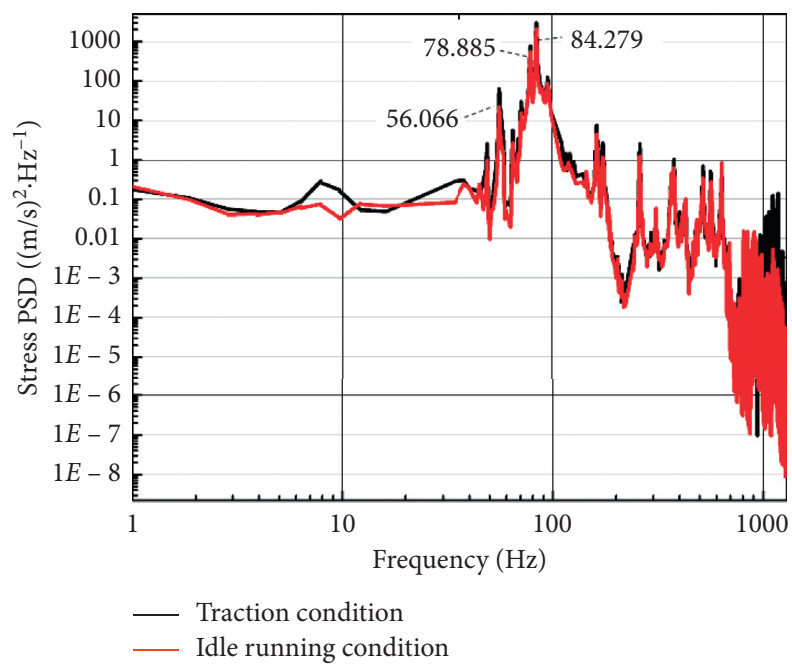

(c)

FIgure 11: (a) PSD of P1 dynamic stress response. (b) PSD of P2 dynamic stress response. (c) PSD of P3 dynamic stress response.

TABLE 2: The peak and frequency of the dynamic stress response at the key nodes.

\begin{tabular}{|c|c|c|c|c|c|c|}
\hline \multirow{2}{*}{ Frequency } & \multicolumn{2}{|c|}{ P1/stress $\left(\mathrm{MPa}^{2} \cdot \mathrm{Hz}^{-1}\right)$} & \multicolumn{2}{|c|}{ P2/stress $\left(\mathrm{MPa}^{2} \cdot \mathrm{Hz}^{-1}\right)$} & \multicolumn{2}{|c|}{ P3/stress $\left(\mathrm{MPa}^{2} \cdot \mathrm{Hz}^{-1}\right)$} \\
\hline & Traction & Idle running & Traction & Idle running & Traction & Idle running \\
\hline 84.279 & 2157.150 & 1479.530 & 578.429 & 396.752 & 3024.340 & 2079.140 \\
\hline 78.885 & 699.092 & 501.669 & 80.046 & 57.410 & 770.571 & 552.419 \\
\hline 56.066 & 366.335 & 128.741 & 12.440 & 6.830 & 62.182 & 21.121 \\
\hline 68.710 & 153.482 & 65.530 & 38.997 & 12.132 & 2.761 & 0.863 \\
\hline 49.225 & 133.416 & 47.614 & 8.123 & 2.918 & 2.397 & 0.926 \\
\hline 261.520 & 87.341 & 16.146 & 1.029 & 0.399 & 2.614 & 0.702 \\
\hline
\end{tabular}

studies show that Dirlik's formulation is more reliable than other fatigue calculation methods with broadband signals $[16,17]$. Dirlik's formulation is written as

$$
D=\frac{E[P] \cdot T}{C} \int_{0}^{\infty} S^{m} p(S) \mathrm{d} S
$$

where $D$ represents the cumulative fatigue damage, $E[P]$ represents the expected number of peaks, $T$ (second) represents the test time, $C$ and $M$ represent the parameters of the S-N curve, $S$ represents the stress range level, and $p(S)$ represents the probability density of $S(\mathrm{MPa})$, which can be expressed as follows: 


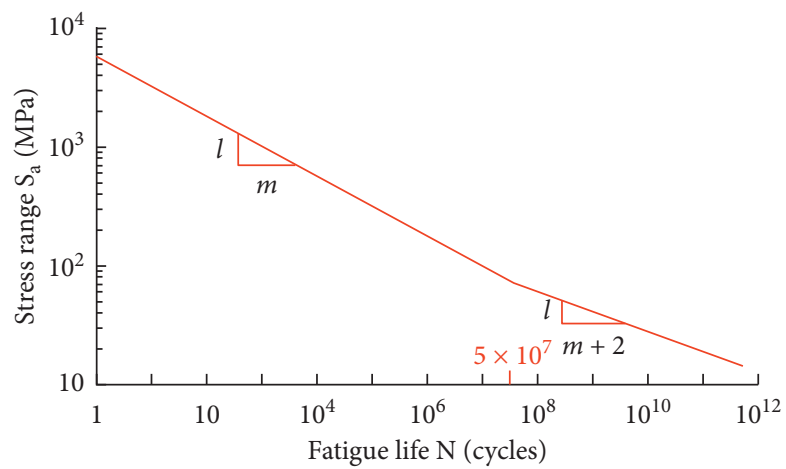

FIgURE 12: S-N curve of $16 \mathrm{MnR}$ steel (95\% reliability).

TABle 3: Comparison of fatigue life under the traction condition and idle running condition.

\begin{tabular}{lccc}
\hline Condition & P1/time $(\mathrm{s})$ & P2/time $(\mathrm{s})$ & P3/time $(\mathrm{s})$ \\
\hline Traction & $10^{7.134}$ & $10^{7.898}$ & $10^{10.012}$ \\
Idle running & $10^{9.523}$ & $10^{10.365}$ & $10^{12.102}$ \\
Life loss (\%) & 99.59 & 99.66 & 99.19 \\
\hline
\end{tabular}

$p(S)=\frac{\left(\left(D_{1} / Q\right) e^{-Z / Q}+\left(D_{2} \cdot Z / R^{2}\right) e^{-z^{2} / 2 \cdot R^{2}}+D_{3} \cdot Z e^{-Z^{2} / 2}\right)}{2 \sqrt{m_{0}}}$

$D_{1}=\frac{2\left(X_{m}-\gamma^{2}\right)}{1+\gamma^{2}}$

$D_{2}=\frac{1-\gamma-D_{1}+D_{1}^{2}}{1-R}$

$D_{3}=1-D_{1}-D_{2}$

$$
Z=\frac{S}{2 \sqrt{m_{0}}}
$$$$
Q=\frac{1.25\left(\gamma-D_{3}-D_{2} R\right)}{D_{1}},
$$$$
R=\frac{\gamma-X_{m}-D_{1}^{2}}{1-\gamma-D_{1}+D_{1}^{2}}
$$$$
\gamma=\frac{m_{2}}{\sqrt{m_{0} m_{4}}}
$$$$
X_{m}=\frac{m_{1}}{m_{0}} \sqrt{\frac{m_{2}}{m_{4}}}
$$

$E[P]=\sqrt{\frac{m_{2}}{m_{4}}}$,

$m_{n}=\int f^{n} \cdot G(f) \mathrm{d} f$, where $m_{n}(n=0,1,2,3,4)$ represents the moment of area of the PSD and $G(f)=2 S(f)$ represents the value of the singleside PSD at frequency $f(\mathrm{~Hz})$.

Based on the Palmgren-Miner rule, assume the sum of damage at fatigue failure is 1 , and total fatigue life $T_{\text {tot }}$ can be solved as follows:

$$
T_{\text {tot }}=\frac{C}{E[P] \cdot \int_{0}^{\infty} S^{m} p(S) \mathrm{d} S} .
$$

4.1. Comparison of Fatigue Life between Traction and Idle Running Conditions. Extract the dynamic stress responses of P1, P2, and P3 calculated in Section 2.4 (Figure 11) and import them into MSC, fatigue software to calculate the fatigue life of the bogie frame. By fully considering the contribution of low-amplitude loads to the fatigue damage, the $\mathrm{S}-\mathrm{N}$ curve of nominal stress associated with $16 \mathrm{MnR}$ steel was extended with a slope of $-1 /(2 m-1)$, starting from the point associated with $5 \times 10^{7}$ times cycles.

According to the BS7608-2015 welding structure fatigue analysis standard, the $\mathrm{S}-\mathrm{N}$ curve level was selected as Class-B to set the parameters, as shown in Figure 12 [18, 19].

As the calculation results are shown in Table 3, by considering the vibration frequency within $1300 \mathrm{~Hz}$, the fatigue life of key node P1 is $10^{9.523}$ (seconds) under the idle running condition; the fatigue life of key node P1 is $10^{7.134}$ (seconds) under the traction condition. Comparing with the idle running condition, the fatigue life of $\mathrm{P} 1, \mathrm{P} 2$, and $\mathrm{P} 3$ under the traction condition is lost about $99.31 \%$ $\left(\left(10^{9.523}-10^{7.134}\right) / 10^{9.523}=99.59 \%\right), 99.66 \%$, and $99.19 \%$, respectively. Therefore, the influence of the motor vibration on the fatigue strength of the metro bogie frame should be fully considered, especially the motor start conditions. 
4.2. Influence of the Vibration with High Frequency and Low Amplitude on Fatigue Damage. It can be seen from Figure 7 that there are some vibrations of high frequency with low amplitude around $1000 \mathrm{~Hz}$, which leads to the low amplitude in the dynamic stress power spectral density of $\mathrm{P} 1$, P2, and P3, as shown in Figure 11. To investigate the influence of dynamic stress of high frequency and low amplitude on the fatigue damage on the bogie frame, the motor vibration frequency within $300 \mathrm{~Hz}$ and $1300 \mathrm{~Hz}$ was selected for fatigue life calculation under the traction condition.

Finally, the results show that the bogie frame can endure 13,614,398 (s) within $300 \mathrm{~Hz}$ and 13,614,444 (s) within $1300 \mathrm{~Hz}$, which is about 46(s) in difference. Because the contribution of the vibration above $300 \mathrm{~Hz}$ to the fatigue damage is too low, its effect can be ignored. The fundamental reason is that the stress response amplitude is too small when the frequency is above $300 \mathrm{~Hz}$.

\section{Conclusion}

According to the random vibration signal of a motor of the vehicle measured on a metro line, the influence of the traction motor vibration on the fatigue life of the bogie frame was studied.

(1) With the motor's traction vibration, the weak positions of fatigue strength are located at the motor seat body, the root of the motor seat base (at the fillet of the midbeam), and the middle of the bottom plate of the side beam (from the fillet with the midbeam). The above three positions are consistent with where the actual fatigue damage appeared.

(2) Mode $7(84.279 \mathrm{~Hz})$ and mode $6(78.885 \mathrm{~Hz})$ with the higher corresponding stress amplitude contribute a lot to the fatigue damage. Besides, mode 7 is excited by the motor's vertical vibration; mode 6 is excited by the motor's transverse vibration.

(3) Compared with the idle running condition, the fatigue life under the traction condition is lost a lot. Therefore, the influence of the motor vibration on the fatigue strength of the metro bogie frame cannot be ignored, especially the motor start conditions. In this case, the contribution of the vibration above $300 \mathrm{~Hz}$ to the fatigue damage is too small, and its effect can be ignored.

\section{Data Availability}

The data used to support the findings of this study are available from the corresponding author upon request.

\section{Conflicts of Interest}

The authors declare that there are no conflicts of interest regarding the publication of this paper.

\section{Acknowledgments}

This research was supported by the National Natural Science Foundation of China (Grant no. 51805373).

\section{References}

[1] Y. Yao, Y. Yan, Z. Hu, and K. Chen, "The motor active flexible suspension and its dynamic effect on the high-speed train bogie," Journal of Dynamic Systems, Measurement, and Control, vol. 140, no. 5, pp. 209-212, 2018.

[2] Y. Yao, X. Zhang, and X. Liu, "The active control of the lateral movement of a motor suspended under a high-speed locomotive," Proceedings of the Institution of Mechanical Engineers, Part F: Journal of Rail and Rapid Transit, vol. 230, no. 6, pp. 1509-1520, 2015.

[3] G. Yang, Y. Wei, G. Zhao et al., "Research on key mechanics of high-speed train," Advances in Mechanics, vol. 45, no. 1, pp. 217-460, 2015.

[4] L. Zhang, Z. S. Ren, S. G. Sun, and G. Yang, "Research on the influence of railway bogie elastic vibration to fatigue life," Railway Locomotive \& Car, vol. 35, no. 2, pp. 115-119, 2018.

[5] B. J. Wang, S. G. Sun, Xi. Wang, Li. Zhang, L. Dong, and C. Y. Jiang, "Research on characteristics of operation loads and fatigue damage of metro train bogie frame," Journal of the China Railway Society, vol. 41, no. 5, pp. 53-60, 2019.

[6] Q. An, F. Li, and M. H. Fu, "Influences of traction motor vibration on bogie frame fatigue strength," Journal of Southwest Jiaotong University, vol. 45, no. 5, pp. 209-212, 2010.

[7] Z. Xia, D. Gong, and J. Zhou, "Decoupling the optimum design of motor suspension system for metro vehicles," Journal of Tongji University, vol. 45, no. 3, pp. 1675-1680, 2017.

[8] M. Zigo, E. Arslan, W. Mack, and G. Kepplinger, "Efficient frequency-domain based fatigue life estimation of spot welds in vehicle components," Forschung im Ingenieurwesen, vol. 83, no. 3, pp. 921-931, 2019.

[9] M. Kassner, "Fatigue strength analysis of a welded railway vehicle structure by different methods," International Journal of Fatigue, vol. 34, no. 2, pp. 103-111, 2012.

[10] W. Tang, W. Wang, Y. Wang, and Q. Li, "Fatigue strength and modal analysis of bogie frame for DMUs exported to Tunisia," Journal of Applied Mathematics and Physics, vol. 2, no. 2, pp. 342-348, 2014.

[11] B. Chen, P. Zhi, and Y. Li, "Fatigue strength analysis of bogie frame in consideration of parameter uncertainty," Frattura Ed Integrità Strutturale, vol. 13, no. 48, pp. 385-399, 2019.

[12] EN13749-2005, Railway Applications Methods of Specifying Structural Requirements of Bogie Frames, British Standards Institution, London, UK, 2005.

[13] S. Li, P. Wu, and Z. Zeng, "Extrapolation of load histories based on kernel density method," Journal of the China Railway Society, vol. 39, no. 7, pp. 25-31, 2017.

[14] UIC615-4-2003, Motive Power Units-Bogie and Running GearBogie Frame Structure Strength Tests, International Union of Railways, France, 2003.

[15] M. Aykan and M. Çelik, "Vibration fatigue analysis and multiaxial effect in testing of aerospace structures," Mechanical Systems and Signal Processing, vol. 23, no. 2, pp. 897-907, 2009.

[16] T. Dirlik, Application of computers in fatigue analysis, Ph.D. thesis, The University of Warwick, Coventry, UK, 1985. 
[17] M. Mršnik, J. Slavic, and M. Boltezar, "Frequency-domain methods for a vibration-fatigue-life estimation- Application to real data," International Journal of Fatigue, vol. 47, no. 2, pp. 8-17, 2013.

[18] British Standards Institution, BS7608 B S Code of Practice for Fatigue Design and Assessment of Steel Structures, British Standards Institution, UK, 2015.

[19] P. Dong and J. Hong, "The master S-N curve approach to fatigue of piping and vessel welds," Weld, vol. 48, no. 2, pp. 28-36, 2004. 